\title{
Update on Heavy Flavor Production in Cold Matter
}

\author{
R. $\operatorname{Vogt}^{1,2, a}$ \\ ${ }^{1}$ Nuclear and Chemical Sciences Division, Lawrence Livermore National Laboratory, \\ Livermore, CA 94550, USA \\ ${ }^{2}$ Physics Department, University of California at Davis, Davis, CA 95616, USA
}

Abstract. I will discuss areas of heavy flavor theory where new progress has been made.

\section{Introduction}

I first focus on open heavy flavor and quarkonium production in $p+p$ collisions before going on to discuss some effects due to cold nuclear matter. The length of a conference proceeding is too short for a complete review of these topics in $p+p$ and $p+A$ interactions. For a somewhat more detailed discussion of open heavy flavor and quarkonium production in $p+p$ collisions, as well as additional references, see Ref. [1].

\section{Open heavy flavor production}

There are currently two approaches to heavy flavor production at colliders: collinear factorization and low $x k_{T}$-factorization approach. I briefly describe each one as applied to single inclusive heavy flavor observables. I then turn to exclusive observables and correlated heavy quark pair production.

There are two main methods of calculating the spectrum of single inclusive open heavy flavor production in perturbative QCD assuming collinear factorization. The underlying idea is similar but the technical approach differs. Both note that large logarithms of $p_{T} / m$ arise at all orders of the perturbative expansion, spoiling the convergence. The first terms in the expansion are the leading (LL) and next-to-leading logarithmic (NLL) terms, $\alpha_{s}^{2}\left[\alpha_{s} \log \left(p_{T} / m\right)\right]^{k}$ and $\alpha_{s}^{3}\left[\alpha_{s} \log \left(p_{T} / m\right)\right]^{k}$ respectively.

It is worth noting that the single inclusive heavy flavor $p_{T}$ distribution is finite at leading order (LO) as $p_{T} \rightarrow 0$ because of the finite quark mass scale. A next-to-leading order (NLO) calculation that assumes production of massive quarks but neglects LL terms, a "massive" formalism, can result in large uncertainties at high $p_{T}[2-4]$. (This massive formalism is sometimes referred to as a fixed-flavor-number (FFN) scheme.) If, instead, the heavy quark is treated as "massless" and the LL and NLL corrections absorbed into the fragmentation functions, the approach breaks down as $p_{T}$ approaches $m$ even though it improves the result at high $p_{T}$. The massless formalism is also sometimes referred to as the zero-mass, variable-flavor-number (ZM-VFN) scheme [5, 6]. There are schemes which interpolate smoothly between the FO/FFN scheme at low $p_{T}$ and the massless/ZMVFN scheme. The fixed-order next-to-leading logarithm (FONLL) approach is one such scheme. In

\footnotetext{
a e-mail: rlvogt@lbl.gov
} 
FONLL, the fixed order and fragmentation function approaches are merged so that the mass effects are included in an exact calculation of the leading $\left(\alpha_{s}^{2}\right)$ and next-to-leading $\left(\alpha_{s}^{3}\right)$ order cross section while also including the LL and NLL terms [7]. The NLO fixed order (FO) result is combined with a calculation of the resumed (RS) cross section in the massless limit. The FO and RS approaches need to be calculated in the same renormalization scheme. The FONLL result is then, schematically,

$$
\mathrm{FONLL}=\mathrm{FO}+(\mathrm{RS}-\mathrm{FOM} 0) G\left(m, p_{T}\right) .
$$

The interpolating function $G\left(m, p_{T}\right) \sim p_{T}^{2} /\left(p_{T}^{2}+(\mathrm{cm})^{2}\right)$ is arbitrary but must approach unity for $m / p_{T} \rightarrow 0$. Fragmentation functions for $D$ and $D^{*}$ mesons have been calculated in an approach consistent with an FONLL calculation [8]. The second interpolation scheme is the generalized-mass variable-flavor-number (GM-VFN) scheme [9]. The large logarithms in charm production for $p_{T} \gg$ $m$ are absorbed in the charm parton distribution function and can thus be evolved by the evolution equations for the parton distributions. The logarithmic terms can be incorporated into the hard cross section to achieve better accuracy for $p_{T} \geq m$. By adjusting the mass-dependent subtraction terms, no interpolating function is required [9].

In the $k_{T}$-factorization approach, off-shell leading order matrix elements for $g^{*} g^{*} \rightarrow c \bar{c}$ are used together with unintegrated gluon densities that depend on the transverse momentum of the gluon, $k_{T}$, as well as the usual dependence on $x$ and $\mu_{F}$. The motivation for choosing the $k_{T}$-factorized approach is that, at sufficiently low $x$, collinear factorization should no longer hold.
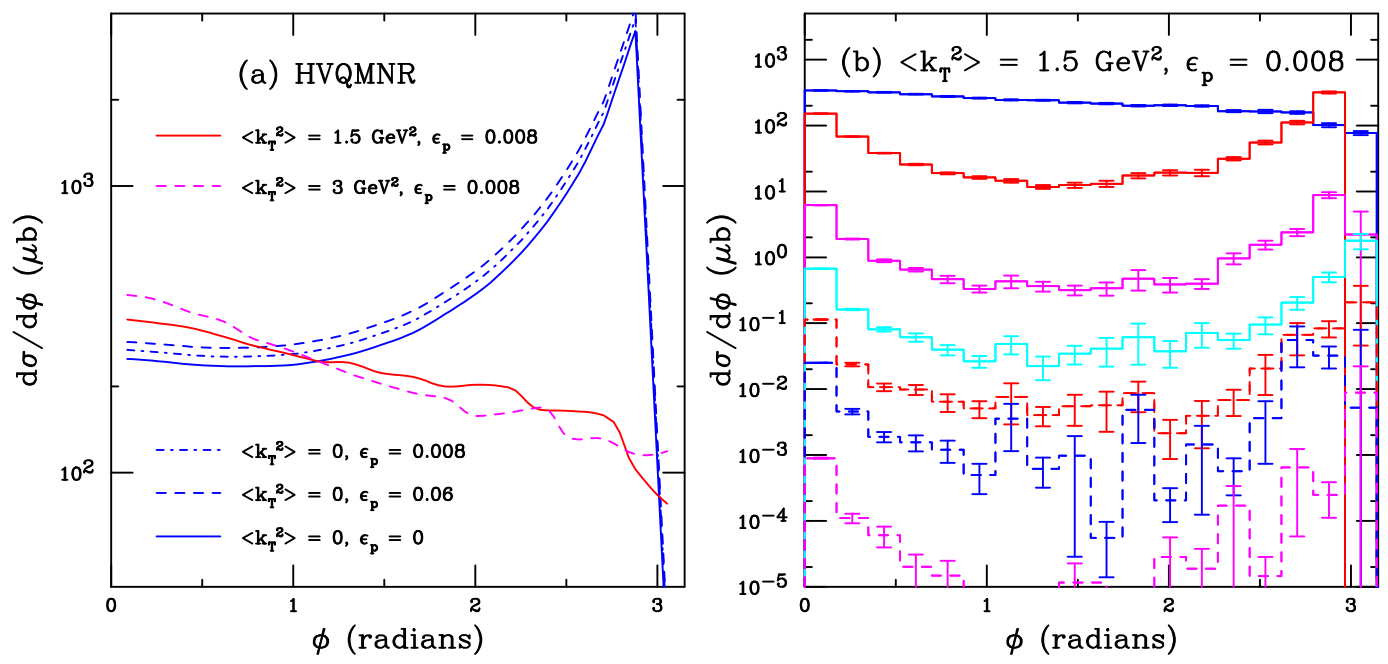

Figure 1. Calculations of the NLO azimuthal distribution between two charm quarks using the HVQMNR code at $\sqrt{s}=7 \mathrm{TeV}$ and forward rapidity. (a) The blue solid, dashed and dot-dashed curves, all without $k_{T}$ broadening, show the effect of modifying the charm fragmentation function. The red solid curve uses a $k_{T}$ broadening consistent with $J / \psi$ production at $7 \mathrm{TeV}$ [14] while the dashed magenta curve shows the effect of doubling the $k_{T}$ broadening. (b) Using $\left\langle k_{T}^{2}\right\rangle=1.5 \mathrm{GeV}^{2}$ and $\epsilon_{p}=0.008$ from the solid red curve in (a), the $p_{T}$ cuts on the charm quarks are varied. From top to bottom the results are: $p_{T}<10 \mathrm{GeV} ; p_{T}>10 \mathrm{GeV} ; p_{T}>20 \mathrm{GeV} ; p_{T}>30 \mathrm{GeV}$; $p_{T}>40 \mathrm{GeV} ; p_{T}>50 \mathrm{GeV} ; p_{T}>75 \mathrm{GeV}$. The curves for all but the lowest $p_{T}$ cut are scaled up by $10^{3}$.

The LHC data has been compared to calculations in both approaches and those assuming collinear factorization compare well with the LHC data. Recent ALICE data [10], at $0<p_{T}<2 \mathrm{GeV}$ supports 
collinear factorization. Their $D^{0}$ results at $7 \mathrm{TeV}$ for $|y|<0.5$, were compared to FONLL, GV-VFNS and LO $k_{T}$-factorization calculations. Only the $k_{T}$-factorized calculation is inconsistent with the shape of the data in the $p_{T}$ range where the calculation should best apply. The forward rapidity data of LHCb at $7 \mathrm{TeV}$ [11] and $13 \mathrm{TeV}$ [12], also agree well with the collinear factorization assumption. The recent $13 \mathrm{TeV}$ data from LHCb is within the uncertainty bands of FONLL and POWHEG (discussed below) down to $p_{T} \rightarrow 0$, albeit with large uncertainty bands and near the upper limit of the band. The GMVFNS calculation is given only for $p_{T}>3 \mathrm{GeV}$ but agrees well with the data with small uncertainties [12]. Perhaps a NLO $k_{T}$-factorized result could lead to improved agreement with the data but so far collinear factorization appears to still work well for low $x$ charm production.

A further challenge to theory may arise from measurements of correlated production, specifically of the azimuthal opening angle of heavy flavors, either by direct reconstruction of both $D$ mesons or of a $D$ meson and the decay product of its partner, either a light hadron or a lepton [13]. Naively, at LO $Q \bar{Q}$ pairs are produced back-to-back with a peak at $\Delta \phi=\pi$. Higher order production will, however, result in a more isotropic distribution in $\Delta \phi$ due to light parton emission in the final state.
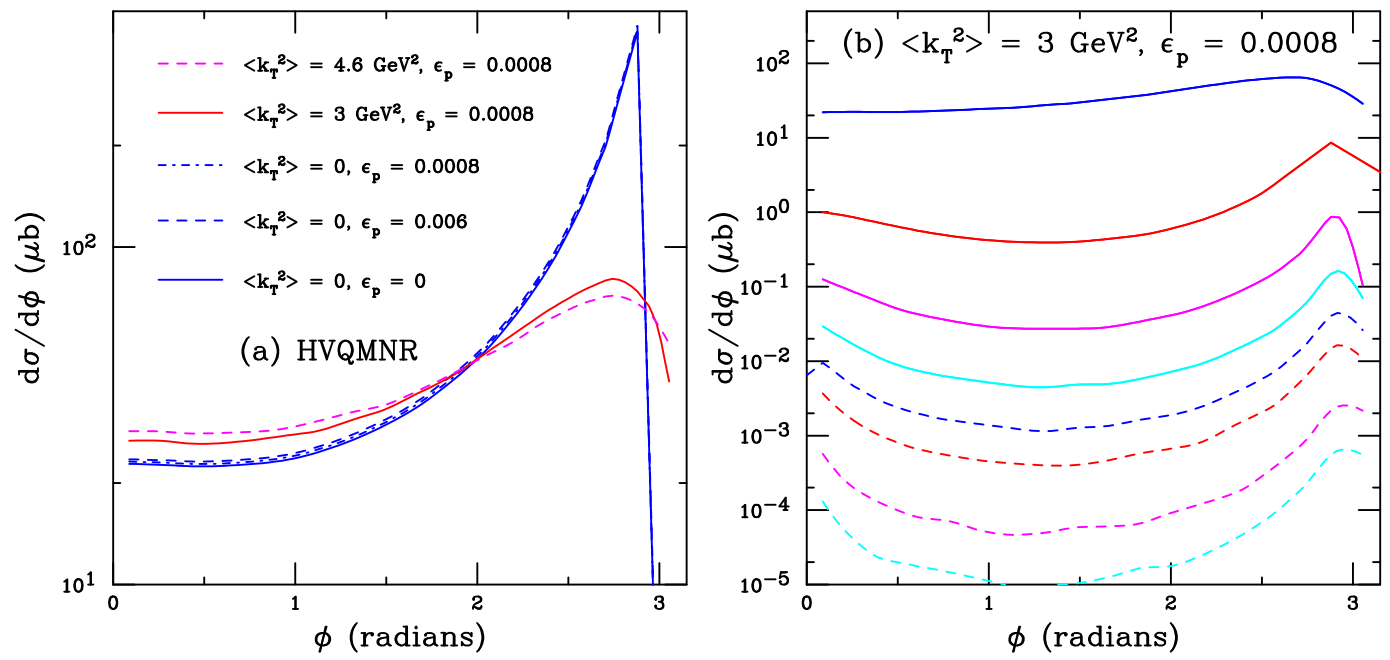

Figure 2. Calculations of the NLO azimuthal distribution between two bottom quarks using the HVQMNR code at $\sqrt{s}=7 \mathrm{TeV}$ and forward midrapidity. (a) The blue solid, dashed and dot-dashed curves, all without $k_{T}$ broadening, show the effect of modifying the charm fragmentation function. The red solid curve uses the $k_{T}$ broadening for $\Upsilon$ production at $7 \mathrm{TeV}$ while the dashed magenta curve shows the effect of doubling the $k_{T}$ broadening. (b) Using $\left\langle k_{T}^{2}\right\rangle=3 \mathrm{GeV}^{2}$ and $\epsilon_{p}=0.0008$ from the solid red curve in (a), the $p_{T}$ cuts on the bottom quarks are varied. From top to bottom the results are: $p_{T}<10 \mathrm{GeV} ; p_{T}>10 \mathrm{GeV} ; p_{T}>20 \mathrm{GeV} ; p_{T}>30 \mathrm{GeV}$; $p_{T}>40 \mathrm{GeV} ; p_{T}>50 \mathrm{GeV} ; p_{T}>75 \mathrm{GeV}$ and $p_{T}>100 \mathrm{GeV}$. No scale factor is included on the results.

The FONLL and GM-VFNS approaches are for single inclusive production only. There are NLO heavy flavor codes that, in addition to inclusive heavy flavor production, also calculate exclusive $Q \bar{Q}$ pair production. HVQMNR [15] uses negative weight events used to cancel divergences numerically. Smearing the parton momentum through the introduction of intrinsic transverse momenta, $k_{T}$, reduces the importance of the negative weight events. HVQMNR does not include any resummation. POWHEG - hvq [16] is a positive weight generator that includes leading-log resummation. The entire event is available since PYTHIA [17] and HERWIG [18] are employed to produce the complete event. In addition to these NLO codes, heavy flavor correlations can also be simulated employing LO event 
generators such as PYTHIA. The $k_{T}$-factorization approach can also be employed to calculate correlated $c \bar{c}$ production since the unintegrated parton densities have a transverse component, giving finite $p_{T}$ and $\Delta \phi$ distributions even at LO.

There are LHC data, particularly from ALICE [19] and LHCb [20], on charm pair correlations to test these approaches.

LHCb measured $c \bar{c}, c c$, and $c J / \psi$ correlations in $p+p$ collisions at $7 \mathrm{TeV}$ for $2<y<4$. About $10 \%$ of the $c \bar{c}$ rate was found in the $c c$ and $c J / \psi$ channels [20]. Only the $c \bar{c}$ pairs can be produced in a single hard scattering. The other events are more consistent with production through double parton scattering. The azimuthal and rapidity distributions for the $c c$ events are consistent with isotropic emission [20], as might be expected from two hard scatterings.

The ALICE collaboration recently presented an analysis of azimuthal correlations between reconstructed $D$ mesons and a light hadron trigger particle [19]. The light hadrons were primary particles, emitted from the collision points. These particles include those from heavy flavor decays, such as the unreconstructed partner $D$ meson. The data were binned according to the transverse momentum of both the $D$ meson and the light hadron. The minimum light hadron $p_{T}$ was soft, $p_{T}>0.3 \mathrm{GeV}$. These data were further subdivided into two $p_{T}$ ranges, $0.3<p_{T}<1 \mathrm{GeV}$ and $p_{T}>1 \mathrm{GeV}$. The $D$ meson $p_{T}$ was considerably higher: $3<p_{T}<5 \mathrm{GeV}, 5<p_{T}<8 \mathrm{GeV}$, and $8<p_{T}<16 \mathrm{GeV}$. To improve statistics, the " $D$ meson" is an average over the $D^{0}, D^{+}$and $D *^{+}$. The ALICE measurements cover the central region, $|y|<0.5$ for the $D$ and $|\Delta \eta|<1$ for the light hadron. The general behavior is, however, the same as the LHCb $D^{0} \bar{D}^{0}$ pairs, a peak at $\Delta \phi=0$ and a smaller enhancement at $\Delta \phi=\pi$. The peak at $\Delta \phi=0$ increases with increasing trigger particle $p_{T}$ and also with increasing $D$ meson $p_{T}$. The data were compared to simulations with various PYTHIA tunes and also POWHEG + PYTHIA. All simulations reproduced the trends of the data [19].

Figure 1(a) shows how the $\phi$ distribution between the $c$ and $\bar{c}$ changes with the fragmentation parameter $\epsilon_{p}$ and the strength of the $k_{T}$ broadening chosen. Note that the NLO result is not a delta function at $\phi=\pi$, as at LO, due to the emission of light partons in the final state, even when $\epsilon_{p}=0$. Changing $\epsilon_{p}$ does not have a strong effect on the shape of $d \sigma / d \phi$. However, introducing $k_{T}$ broadening changes $d \sigma / d \phi$ from peaked at $\phi \sim \pi$ to more isotropic with a maximum at $\phi=0$ instead. No $p_{T}$ cut is made on the momentum of the charm quarks. The effect of a $p_{T}$-dependent cut on the charm quarks is explored in Fig. 1(b) using $\left\langle k_{T}^{2}\right\rangle=1.5 \mathrm{GeV}^{2}$, consistent with the broadening needed to describe the $J / \psi p_{T}$ dependence in Ref. [14], and $\epsilon_{p}=0.008$, softer than the traditional Peterson function. With these choices, the HVQMNR $p_{T}$ distribution is in agreement with the FONLL $D^{0} p_{T}$ distribution for the same choice of charm mass and scale parameters. When only low $p_{T}$ charm quarks are considered, $p_{T}<10 \mathrm{GeV}$, the azimuthal distribution is rather flat. Increasing the $p_{T}$ cut on the charm quarks results in distributions that are peaked at $\phi=0$ and $\pi$ : either the charm quarks are aligned opposite a high momentum light parton $(\phi=0)$ or the charm quarks are effectively back-to-back with a soft light parton emitted $(\phi \sim \pi)$.

Figure 2 shows a similar calculation for bottom quarks. The effect of changing the fragmentation function is reduced for the heavier bottom quarks, as is the effect of $k_{T}$ broadening. The harder $p_{T}$ dependence for $b$ quarks allows the azimuthal distribution to be calculated at higher $p_{T}$, up to $p_{T}>100 \mathrm{GeV}$ with higher statistics. The same trends are observed for bottom and charm quarks.

It appears that, so far, the open charm results presented by the LHC collaborations, both single inclusive production and charm pair correlations, are in agreement with calculations based on collinear factorization with single hard scatterings. The exception, the $c c$ and $c J / \psi$ events at LHCb, are consistent with double parton scattering. 


\section{Quarkonium Production}

I now turn to quarkonium production. Perturbative QCD is employed to calculate the production characteristics. A number of models have been used since the discovery of the $J / \psi$ just over 40 years ago. One of the first was the color evaporation model (CEM) [21-23] which does not specify the color or spin state of the produced $Q \bar{Q}$ pairs. The next was the color singlet model (CSM) [24] which only considered quarkonium states produced as color neutral objects. Despite the addition of a number of higher order corrections, a good description of the data has not been achieved. The current most successful approach, non-relativistic QCD (NRQCD) [25, 26], is based on an expansion of the cross section in both the strong coupling constant and $Q \bar{Q}$ velocity with a separation of the hard and soft scales so that contributions to the cross section are divided into different color states with different weight factors, long distance matrix elements (LDMEs), assumed to be universal, which are adjusted to data. Most calculations have been done in the collinear factorization approach. However, some work has also been done in $k_{T}$-factorization both in the context of the CSM and NRQCD. In the remainder of this section, I will discuss only recent results in NRQCD and the CEM.

According to the NRQCD factorization theorem for $J / \psi$ production, the production cross section can be written as

$$
\sigma_{J / \psi}=\Sigma_{n} \sigma_{c \bar{c}[n]}\left\langle O^{J / \psi}[n]\right\rangle
$$

where the sum over $n$ includes all Fock states, including color octet states [25]. The cross section $\sigma_{c \bar{c}[n]}$ is the production rate of a $c \bar{c}$ pair in the color and spin state $n$, calculated in perturbative QCD. Finally, $\left\langle O^{J / \psi}[n]\right\rangle$ represents the LDMEs which describe the conversion of the $c \bar{c}[n]$ state into a final state $J / \psi$, assuming that the hadronization does not change the spin or momentum. The full cross section includes convolution with the parton densities appropriate to the process in question.

In comparisons of the NRQCD calculations to data, the color octet LDMEs are determined above some $p_{T}$ cut, along with uncertainty on their values. The uncertainty band is determined by fixing the LDMEs to their central fit values and varying the renormalization and factorization scales by a factor of two relative to the central values of $\mu_{R}=\mu_{F}=m_{T}=\left(p_{T}^{2}+4 m_{c}^{2}\right)^{1 / 2}$ with $m_{c}=1.5 \mathrm{GeV}$. No variation of the charm quark mass or the LDMEs are included in the uncertainty band [26].

One of the most important questions for NRQCD to resolve is whether or not the color octet LDMEs are indeed universal. Their universality has been called into increasing question recently. Different groups arrive at different values of the LDMEs depending on the chosen $p_{T}$ cut, the data sets included in the analysis, and whether or not the polarization data are included in the fits, see Ref. [26] and references therein. In addition, the LDMEs fit to collider data do not agree with the integrated cross sections, $d \sigma /\left.d y\right|_{y=0}$ [27]. Recent measurements of the $\eta_{c}$ at forward rapidity in $\mathrm{LHCb}$ [28] strongly disagree with the $p_{T}$ distributions calculated assuming heavy quark spin symmetry [29].

If one goes beyond current next-to-leading order (NLO) calculations, it is not clear that the $p_{T}$ dependence would substantially change. The $p_{T}$ distributions already include the powers giving the hardest $p_{T}$ distributions. Adopting more fit parameters, including the mass and scales, will not have a strong effect on the $p_{T}$ distributions. Changing the factorization scale has the largest effect on the shape but modifying the renormalization scale or the quark mass has a larger effect on the magnitude than the shape of the distributions.

It has been suggested that NRQCD factorization does not hold for polarization. However, if factorization holds for the $p_{T}$ distributions, then, under the assumption that the spin and momentum are unaffected by hadronization, the initial polarization should survive as well.

The difficulties that have plagued the NRQCD description of $J / \psi$ production are reduced for $\Upsilon$ production. The larger mass, higher scale and lower velocity could make the $\Upsilon$ a better candidate for an accurate description by NRQCD. In addition, since there are more $\Upsilon$ states, there are more 
color octet LDMEs available for fitting, allowing a description of the $p_{T}$-dependent yields and the polarization simultaneously.

Some new developments to the color evaporation model make it worth a second look. In the CEM, heavy flavor and quarkonium production is treated on an equal footing. In the CEM, the quarkonium production cross section is some fraction of all $Q \bar{Q}$ pairs below the $H \bar{H}$ threshold where $H$ is the lowest mass heavy-flavor hadron. Thus the CEM cross section is simply the $Q \bar{Q}$ production cross section with a cut on the pair mass. The color and spin have been integrated over so that the color of the state is said to have been 'evaporated' away without changing the kinematics of the pair.
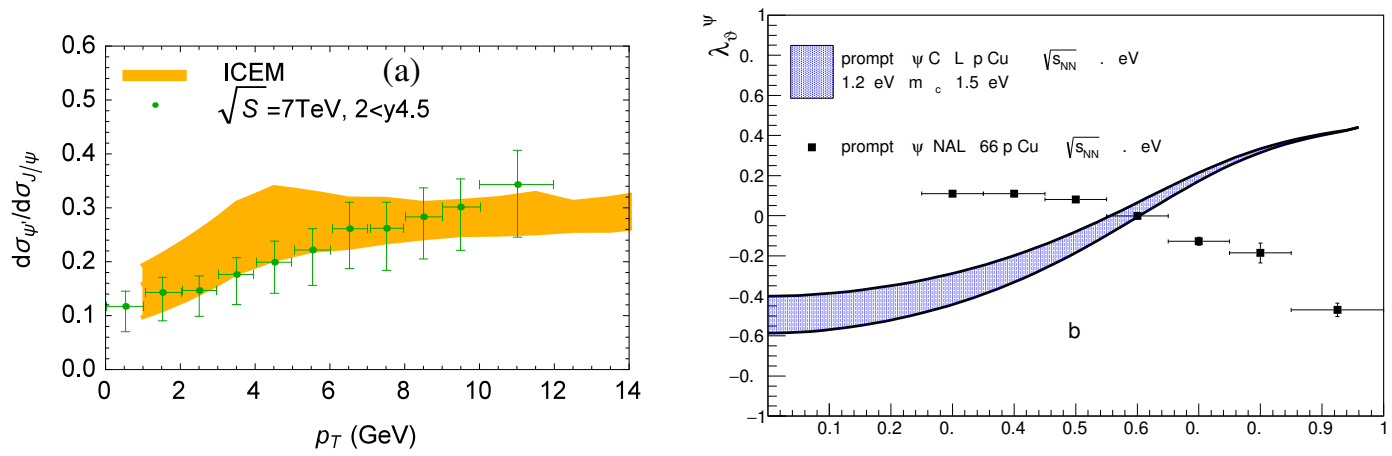

Figure 3. (a) Results for ratio of the $\psi^{\prime}$ to $J / \psi$ production cross sections at forward rapidity for $7 \mathrm{TeV} p+p$ collisions [30]. (b) The $x_{F}$ dependence of the polarization parameter $\lambda_{\theta}$ for prompt production of $J / \psi$ in $p+\mathrm{Cu}$ collisions at $\sqrt{s_{N N}}=38.8 \mathrm{GeV}$ is compared to the E866/NuSea data. The horizontal uncertainties are the experimental bin widths. From [31].

At leading order, the production cross section of quarkonium state $C$ in a $p+p$ collision is

$$
\sigma_{C}^{\mathrm{CEM}}\left(s_{N N}\right)=F_{C} \sum_{i, j} \int_{M^{2}}^{4 m_{H}^{2}} d \hat{s} \int d x_{1} d x_{2} f_{i}^{p}\left(x_{1}, \mu_{F}^{2}\right) f_{j}^{p}\left(x_{2}, \mu_{F}^{2}\right) \mathcal{J}(\hat{s}) \hat{\sigma}_{i j}\left(\hat{s}, \mu_{F}^{2}, \mu_{R}^{2}\right),
$$

where $i j=q \bar{q}$ or $g g$ and $\hat{\sigma}_{i j}(\hat{s})$ is the $i j \rightarrow Q \bar{Q}$ subprocess cross section. Here $\mathcal{J}(\hat{s})$ is a kinematicsdependent Jacobian. In the traditional CEM, the lower limit on the integration over $\hat{s}$ is $M^{2}=4 m_{c}^{2}$. The fraction $F_{C}$ must be universal so that, once it is fixed by data, the quarkonium production ratios should be constant as a function of $\sqrt{s}, y$ and $p_{T}$. The actual value of $F_{C}$ depends on the heavy quark mass, $m$, the scale parameters, the parton densities and the order of the calculation. The same values of the charm quark mass and scale parameters as found in Ref. [14] are employed to obtain the normalization $F_{C}$ for the $J / \psi$,

Two recent updates of the CEM are worth noting. The first is a calculation of the $\psi^{\prime} / \psi$ ratio as a function of $p_{T}$ in an "improved" CEM calculation (ICEM) [30]. In this calculation, the $p_{T}$ of the quarkonium state is modified by the ratio of the pair invariant mass to the quarkonium mass, $M_{C}$. In addition, the lower limit on the integral is now $M^{2}=M_{C}^{2}$. Thus a rise in the ratio with $p_{T}$ consistent with measurement is found [30], see Fig. 3(a). The second is the first calculation of polarization in the CEM. If one assumes that the spin of the quarks are either aligned or anti-aligned with the momentum, $J_{z}=-1,0$ or 1 , and this alignment, like the momentum of the quarks, survives hadronization, the polarization can be obtained. The first calculation separated the longitudinal and transverse components [32] while the second separated the $\mathrm{S}$ and $\mathrm{P}$ states to calculate the polarization parameter, $\lambda_{\psi}$ 
in the ICEM [31]. The results so far are at LO. Therefore, only comparison to fixed-order results is possible so far, as shown for $p+\mathrm{Cu}$ collisions at $\sqrt{s}=38.8 \mathrm{GeV}$. Work is underway to calculate the $p_{T}$ dependence in the $k_{T}$-factorization approach before attempting a full NLO calculation.

\section{Heavy Flavors in Cold Nuclear Matter}
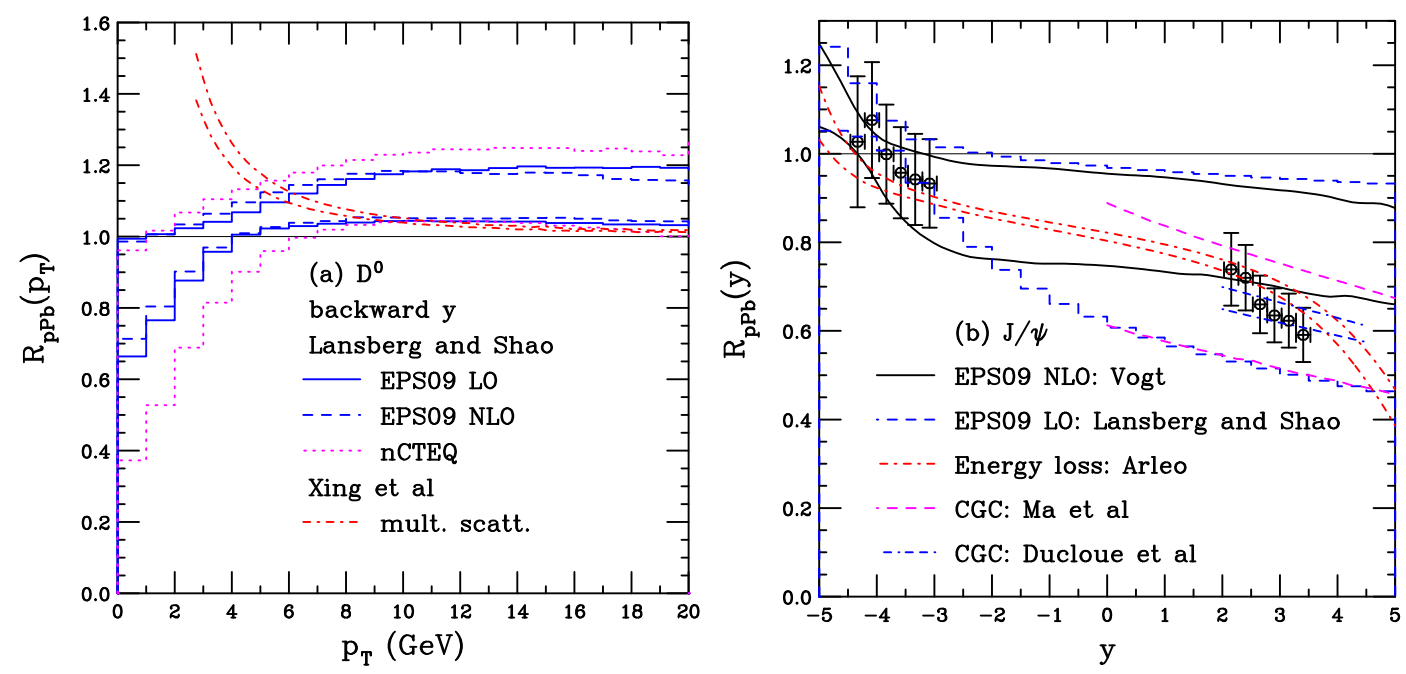

Figure 4. (a) The $D^{0}$-meson $R_{p \mathrm{~Pb}}\left(p_{T}\right)$ at $-4<y<-2.96$. The red band is by Xing et al.. The data-driven shadowing results of Lansberg and Shao at $-4.46<y<-2.96$ are shown as histograms for EPS09 NLO (solid blue), EPS09 LO (dashed blue) and nCTEQ15 (dotted magenta). (b) The $J / \psi R_{p \mathrm{~Pb}}(y)$ for EPS09 NLO by Vogt (black), the data-driven result from Lansberg and Shao for EPS09 LO (blue dashed histogram), the energy loss calculation by Arleo (dot-dashed red), and the CGC calculations by Doucloué et al. (blue dot-dashed) and Ma et al. (dashed magenta). The ALICE data [34] are shown in black. For details, see Albacete et al. [35].

There are several important cold nuclear matter (CNM) effects that need to be taken into account when determining the strength of deconfinement effects on a particular final state. These include modifications of the parton distribution functions in nuclei, either by shadowing in collinear factorization or through the color glass condensate (CGC) approach; energy loss and/or $k_{T}$ broadening in the Cronin effect; and isospin effects, negligible for heavy flavor production. In addition, for quarkonium effects such as nuclear absorption and comover breakup may play a role. See Ref. [35] for a summary of these effects as well as predictions for the $8 \mathrm{TeV}$ LHC $p+\mathrm{Pb}$ run and Refs. [33] for a summary for $5 \mathrm{TeV}$ predictions as well as comparison to data.

Figure 4 shows results for (a) $D^{0}$ and (b) $J / \psi$ modifications in $p+\mathrm{Pb}$ relative to $p+p$ collisions at 8 $\mathrm{TeV}$. The multiple scattering model of $D^{0}$ modifications gives a significantly different ratio $R_{p \mathrm{~Pb}}\left(p_{T}\right)$ at low $p_{T}$ than the results with shadowing alone: one produces an enhancement while the other gives effective suppression. The $J / \psi$ results as a function of rapidity are compared to preliminary ALICE data [34]. All the models give reasonable agreement with the data so far. Note that the CGC predictions are only shown at forward rapidity. 


\section{Acknowledgments}

I thank the organizers for the invitation to give this talk. This work was performed under the auspices of the U.S. Department of Energy by Lawrence Livermore National Laboratory under Contract DEAC52-07NA27344 and supported by the U.S. Department of Energy, Office of Science, Office of Nuclear Physics (Nuclear Theory) under contract number DE-SC-0004014.

\section{References}

[1] R. Vogt, EPJ Web Conf. 137 (2017) 01022.

[2] P. Nason et al., Nucl. Phys. B 303 (1988) 607; B 327 (1988) 49; B 335 (1990) 260(E).

[3] W. Beenakker, H. Kuijf, W. L. van Neerven and J. Smith, Phys. Rev. D 40, (1989) 54.

[4] W. Beenakker et al., Nucl. Phys. B 351 (1991) 507.

[5] M. Cacciari and M. Greco, Nucl. Phys. B 421 (1994) 530.

[6] J. Binnewies, B. A. Kniehl and G. Kramer, Phys. Rev. D 58 (1998) 014014.

[7] M. Cacciari, M. Greco and P. Nason, JHEP 05 (1998) 007.

[8] M. Cacciari and P. Nason, JHEP 0309 (2003) 006.

[9] B. A. Kniehl, G. Kramer, I. Schienbein and H. Spiesberger, Phys. Rev. D 71 (2005) 014018.

[10] J. Adam et al. [ALICE Collaboration], Phys. Rev. C 94 (2016) 054908.

[11] R. Aaij et al. (LHCb Collaboration), Nucl. Phys. B 871 (2013) 1.

[12] R. Aaij et al. [LHCb Collaboration], JHEP 1603 (2016) 159, Erratum: [JHEP 1609 (2016) 013], Erratum: [JHEP 1705 (2017) 074]

[13] A. Mischke, Phys. Lett. B 671 (2009) 361.

[14] R. E. Nelson, R. Vogt and A. D. Frawley, Phys. Rev. C 87 (2013) 014908.

[15] M. L. Mangano, P. Nason, and G. Ridolfi, Nucl. Phys. B 373 (1992) 295.

[16] S. Frixione, P. Nason, and G. Ridolfi, JHEP 0709 (2007) 126; arXiv:0707.3081 [hep-ph].

[17] T. Sjostrand et al., Comput. Phys. Commun. 135 (2001) 238; arXiv:hep-ph/0308153.

[18] G. Corcella et al., JHEP 0101 (2001) 010.

[19] J. Adam et al. [ALICE Collaboration], Eur. Phys. J. C 77 (2017) 245.

[20] R. Aaij et al. [LHCb Collaboration], JHEP 1206 (2012) 141, [JHEP 1403 (2014) 108].

[21] R. Gavai et al., Int. J. Mod. Phys. A 10 (1995) 3043.

[22] G. A. Schuler and R. Vogt, Phys. Lett. B 387 (1996) 181.

[23] J. F. Amundson, O. J. P. Eboli, E. M. Gregores and F. Halzen, Phys. Lett. B 390 (1997) 323.

[24] R. Baier and R. Rückl, Z. Phys. C 19 (1983) 251.

[25] G. T. Bodwin, E. Braaten and G. P. Lapage, Phys. Rev. D 51 (1995) 1125.

[26] N. Brambilla et al., Eur. Phys. J. C 74 (2014) 2981.

[27] Y. Feng, J. P. Lansberg and J. X. Wang, Eur. Phys. J. C 75 (2015) 313.

[28] R. Aaij et al. [LHCb Collaboration], Eur. Phys. J. C 75 (2015) 311.

[29] M. Butenschön, Z.-G. He and B. A. Kniehl, Phys. Rev. Lett. 114 (2005) 092004.

[30] Y. Q. Ma and R. Vogt, Phys. Rev. D 94 (2016) 114029.

[31] V. Cheung and R. Vogt, Phys. Rev. D 96 (2017) 054014.

[32] V. Cheung and R. Vogt, Phys. Rev. D 95 (2017) 074021.

[33] J. L. Albacete et al., Int. J. Mod. Phys. E 25 (2016) 1630005.

[34] ALICE Collaboration, CERN-ALICE-PUBLIC-2017-001.

[35] J. L. Albacete et al., arXiv:1707.09973 [hep-ph]. 